\title{
Associação entre transtornos mentais comuns e o nível de atividade física em estudantes universitárias
}

\section{Association between common mental disorders and the level of physical activity in university students}

\author{
Laís Rodrigues Monteiro, Rosa Leocadio Munhoz, Simone Lara
}

Como citar este artigo: MONTEIRO, Laís R.; MUNHOZ,

Rosa L.; LARA, Simone. Associação entre transtornos mentais comuns e o nível de atividade física em estudantes universitárias. Revista Saúde (Sta. Maria). 2019; 45 (3)

\section{Autor correspondente:}

Nome: Simone Lara

E-mail: simonelara@unipampa.

edu.br

Telefone: (55) 34|3-432|

Formação Profissional: Doutora

em Educação em ciências:

química da vida e saúde pela

Universidade Federal de Santa

Maria, Santa Maria, RS, Brasil.

Filiação Institucional: Universidade Federal do Pampa (UNIPAM-

PA); Faculdade de Ciências da

Saúde

Endereço para correspondência:

Campus Uruguaiana, BR 472, km

592, CP 118

Cidade: Uruguaiana

Estado: Rio Grande do Su

CEP: 97500-970

Data de Submissão:

09/04/2019

Data de aceite:

04/08/20 19

Conflito de Interesse: Não há conflito de interesse

\section{(c) $\mathrm{BY}-\mathrm{NC}-\mathrm{ND}$}

\section{RESUMO}

Objetivo: Identificar a associação entre transtornos mentais comuns (TMC) e o nível de atividade física habitual (NAF) em estudantes universitárias. Métodos: Este estudo transversal incluiu 21 acadêmicas, submetidas a um protocolo de avaliação, por meio do questionário Self-Report Questionnaire (SRQ$20)$ e da avaliação do NAF. As jovens foram classificadas entre mais ativas (>6.000 passos/dia) e menos ativas (<6.000passos/dia). Resultados: Não houve associação entre os TMC e o NAF nesta amostra de estudantes analisadas ( $p>0,05)$. Porém este estudo demonstrou um perfil preocupante, relacionado ao baixo NAF (5.318,14 passos por dia) e uma pontuação indicativa de TMC $(9,04$ no SRQ20), indicando dois fatores de risco que podem afetar a qualidade de vida dessas jovens. Conclusão: Com base nos dados obtidos, são necessárias estratégias para incentivar a prática de atividade física no ambiente acadêmico, melhorando a saúde e o bem-estar físico e mental dos jovens.

PALAVRAS-CHAVE: Saúde Mental; Estudantes; Transtornos Mentais; Atividade Física.

\section{ABSTRACT}

Objective: To identify the association between common mental disorders (CMD) and the level of habitual physical activityin (HPA) in college students. Methods: This cross-sectional study included 21 students, in which they were submitted to an evaluation protocol, through the Self-ReportQuestionnaire (SRQ-20), and HPA. The young women were classified as more active (>6,000 steps / day) and less active ( $<6,000$ steps / day). Results: There was no association between CMD and NAF in this sample of students analyzed $(p>0.05)$. However, this study showed a worrying profile, related to the low level of physical activity (5,318.14 steps per day) and an indicative score of CMD (9.04 in SRQ-20), indicating two risk factors that may affect the quality of life of these young women. Conclusions: Based on the data obtained, strategies are needed to encourage the practice of physical activity in the academic environment, improving the health and physical and mental well-being of young people.

KEYWORDS: Mental Health; Students; Mental Disorders; Physical activity. 


\section{INTRODUÇÃO}

O ingresso ao ensino superior corresponde ao primeiro projeto de vida e profissional de muitos jovens, contudo pode ser gerador de crises e de desafios pessoais, interpessoais, familiares e institucionais. Tais desafios estão atrelados à adaptação à instituição e ao curso, níveis maiores de autonomia na aprendizagem, novas exigências cognitivas e de estudo, gestão do tempo e do dinheiro, afastamento dos amigos e dos familiares, relacionamento com os professores e os colegas de classe, tipo de moradia, e perspectivas com a carreira'.

Este contexto pode levar ao desenvolvimento de fatores de risco para afecções psicológicas, corroborando com os achados de Ansolin et al. ${ }^{2}$ e Pinho ${ }^{3}$. Ansolin et al. ${ }^{2}$ identificaram, em uma amostra de acadêmicos da área da saúde, sintomas associados à fadiga, as sensações de sentir-se nervoso, tenso ou preocupado; ter sensação de tristeza; dentre outros. Já Pinho ${ }^{3}$ encontrou queixas associadas à déficits de habilidades sociais, seguidas do transtorno depressivo e transtorno de ansiedade, em uma amostra de acadêmicas jovens, de cursos da área da saúde.

Cabe destacar que esses sintomas podem estar associados ao desenvolvimento dos transtornos mentais comuns (TMC), que se caracterizam por sintomas como insônia, fadiga, irritabilidade, esquecimento, dificuldade de concentração e queixas somáticas ${ }^{4}$. Segundo o caderno de Atenção Básica de Saúde Mental do Ministério da Saúde ${ }^{5}$, diversos fatores somados podem resultar no sofrimento mental comum, tais como situação de vulnerabilidade (gênero, pobreza, cor da pele e desigualdade), desestabilização (eventos de vida e seus significados, como perda de parentes, relações abusivas e violência), o temperamento ou personalidade e baixa autoestima.

Vale destacar que a entrada do jovem na universidade é um período marcante devido a novas relações sociais e um novo estilo de vida, trazendo consigo alterações de condutas de saúde, dentre elas a diminuição do nível de atividade física. Este fator, que já é um fator de risco para uma série de disfunções, também está associado com os TMC, uma vez que o estudo de Adamoli e Azevedo ${ }^{6}$ identificou uma baixa prevalência de atividade física e de lazer em sujeitos com diagnóstico de TMC.

Assim, com base no exposto, o objetivo desse estudo foi identificar a associação entre os TMC e o nível de atividade física habitual (NAF) em estudantes universitárias do curso de Fisioterapia.

\section{MÉTODO}

Esse estudo transversal, descritivo e quantitativo, incluiu uma amostra de conveniência, formada por mulheres, de 19 a 39 anos, universitárias do curso de Fisioterapia da Universidade Federal do Pampa, que já tenham cursado, no mínimo, 04 semestres do curso. Cabe destacar que foram escolhidas apenas mulheres, uma vez que o curso selecio- 
nado apresenta um expressivo número de acadêmicas do sexo feminino. Os critérios de exclusão adotados no presente estudo foram: acadêmicas que estivessem em afastamento da universidade por motivo de saúde ou que apresentassem diagnóstico de TMC, atestado por laudo médico. Os critérios éticos foram adotados, e o estudo foi aprovado pelo Comitê de Ética institucional sob o número 1.694.108, e os participantes assinaram o termo de consentimento livre e esclarecido (TCLE).

O protocolo de avaliação utilizado foi composto por:

a) Questionário de Saúde - construído pelos pesquisadores, incluindo dados de identificação, questões relacionadas à saúde, em relação ao uso de medicações, lesões musculoesqueléticas prévias, tabagismo e processo de emagrecimento;

b) Avaliação antropométrica - através da mensuração da massa corporal e da estatura;

c) Instrumento SRQ-20 (Self-Report Questionnaire)- utilizado para identificar distúrbios psiquiátricos em nível de atenção primária, desenvolvido por Harding et al. ${ }^{7}$ e validado no Brasil por Mari e Williams ${ }^{8}$. É composto por 20 questões com respostas "sim" ou "não", sendo 4 perguntas sobre sintomas físicos e 16 sobre desordens psicoemocionais. A cada resposta "sim" foi atribuído um ponto, resultando em uma pontuação final que varia de 0 a 20 pontos ${ }^{8}$, sendo que a presença de oito ou mais respostas positivas para ambos os sexos, é indicativa de TMC ${ }^{9}$. Sendo assim, optou-se por estratificar os sujeitos em duas categorias: com risco para TMC ( $\geq 8$ questões) e sem risco para TMC (<8 questões).

d) Avaliação do nível de atividade física - através do uso de um pedômetro digital (BP, Techline, Brasil), instrumento que registra o número de passos diários, a fim de identificar o NAF dos sujeitos através do deslocamento (passos/dia) pelas oscilações verticais do corpo ${ }^{10} .0$ instrumento foi entregue para as acadêmicas, configurado individualmente (de acordo com a massa corporal e a distância do passo), juntamente com uma ficha de registro do número de passos, a fim de que registrassem seus passos por dia, com orientações sobre o seu uso adequado. As mesmas foram orientadas a utilizar o pedômetro ao acordar, permanecendo todo o dia com o aparelho, preso à cintura, retirando-o ao final do dia, antes de deitar, durante 7 dias consecutivos. No final do sétimo dia, os pedômetros foram entregues às pesquisadoras, juntamente com a ficha de registro de passos, devidamente preenchida. Esse procedimento está em conformidade com as orientações de Bassett et al. ${ }^{11}$, ressaltando que as medições devem ser feitas durante 3-7 dias para obter estimativas válidas e confiáveis.

Para estratificar a atividade física das participantes da pesquisa, com relação ao uso do pedômetro, estratificamos as estudantes entre mais ativas (>6.000 passos/dia) e menos ativas (<6.000passos/dia), conforme o estudo de Lara et al. ${ }^{12}$, utilizado para amostras menores, em jovens do sexo feminino.

Para a análise dos dados foi utilizada estatística descritiva, por meio de medidas de média, desvio padrão e frequências. Para a normalidade dos dados foi utilizado o teste de Kolgomorov-Smirnov que indicou que os dados pos- 
suem uma distribuição normal. $O$ teste "t" para amostras independentes foi utilizado para comparação entre grupos, e a correlação de Pearson foi utilizada para verificar a associação entre o NAF e os TCM. Foi considerado para todas as análises um nível de significância de p>0,05.

\section{RESULTADO}

Foram incluídas 21 acadêmicas, sendo que as características gerais da amostra, incluindo idade, dados antropométricos, número de passos diários e valores do SRQ-20 estão presentes na tabela 1. Ademais, 5 relatam uso de medicação atual, 3 sofreram lesões prévias, 1 é tabagista e 2 realizaram tratamento para emagrecimento prévio.

Tabela 1. Características gerais da amostra

\begin{tabular}{ll}
\hline Variável & Valores \\
\hline Idade (anos) & $24,67 \pm 3,78$ \\
Massa $(\mathrm{Kg})$ & $64,52 \pm 12,50$ \\
Estatura $(\mathrm{m})$ & $1,63 \pm 0,06$ \\
Número de passos & $5318,14 \pm 2446,71$ \\
SRQ-20 & $9,04 \pm 4,14$ \\
\hline
\end{tabular}

Análise descritiva, com média e DP \pm

A figura 1 apresenta a correlação entre o NAF e a Pontuação do SRQ-20 das acadêmicas avaliadas. A análise identificou que houve uma associação negativa, porém não significativa entre as variáveis.

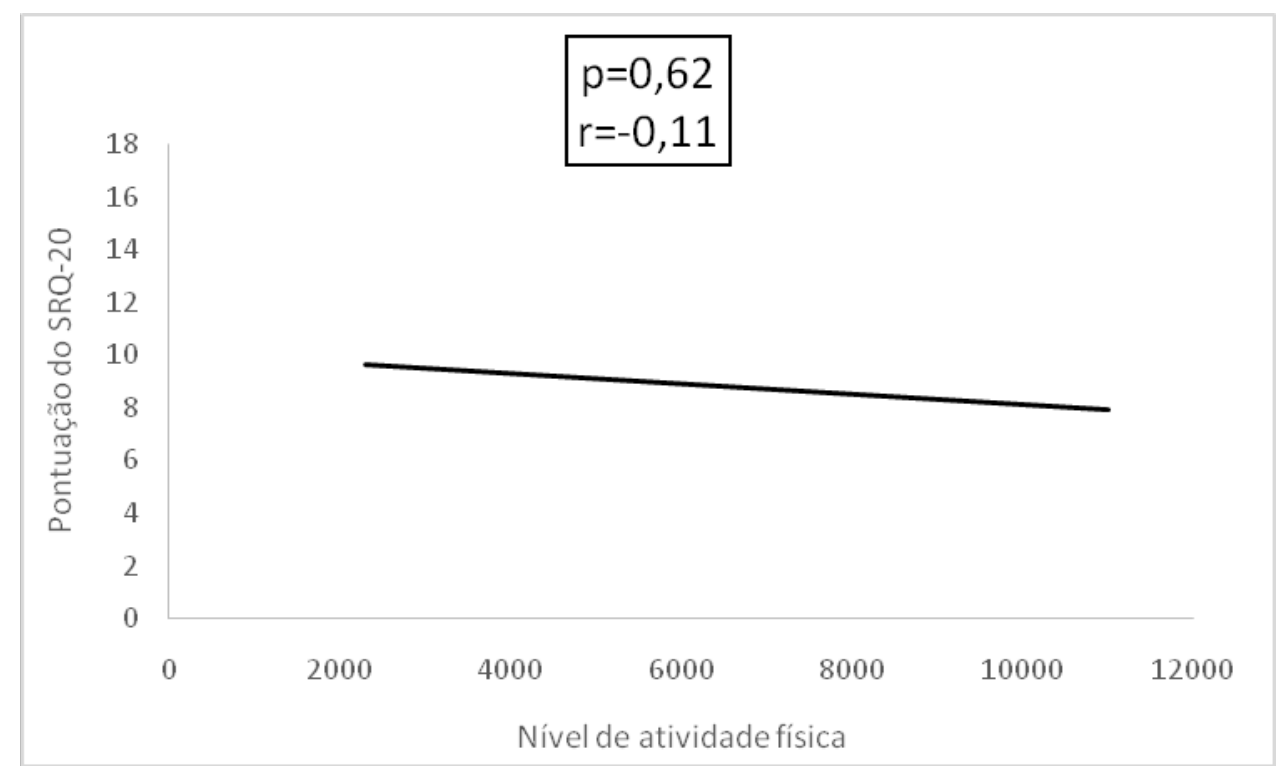

Figura 1. Associação entre o NAF e a Pontuação do SRQ-20 
A figura 2 apresenta uma análise comparativa da pontuação do SRQ-20 entre os sujeitos mais ativos (>6.000 passos/dia) e menos ativos (<6.000 passos/dia), no qual não foram observadas diferenças significativas entre os grupos.

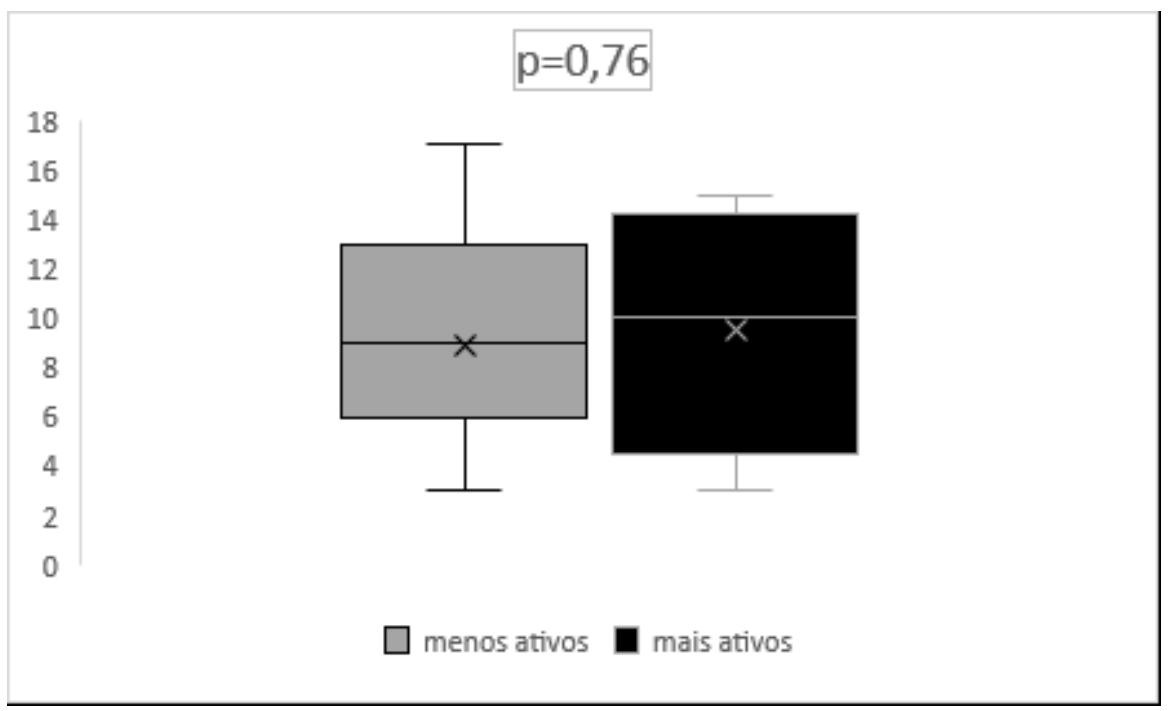

Figura 2. Comparação da pontuação do $S R Q-20$ entre mais ativas e menos ativas

Da mesma forma, não houve diferença significativa do NAF entre os sujeitos com risco para TMC ( $\geq 8$ questões) e sem risco para TMC (<8 questões) (figura 3).

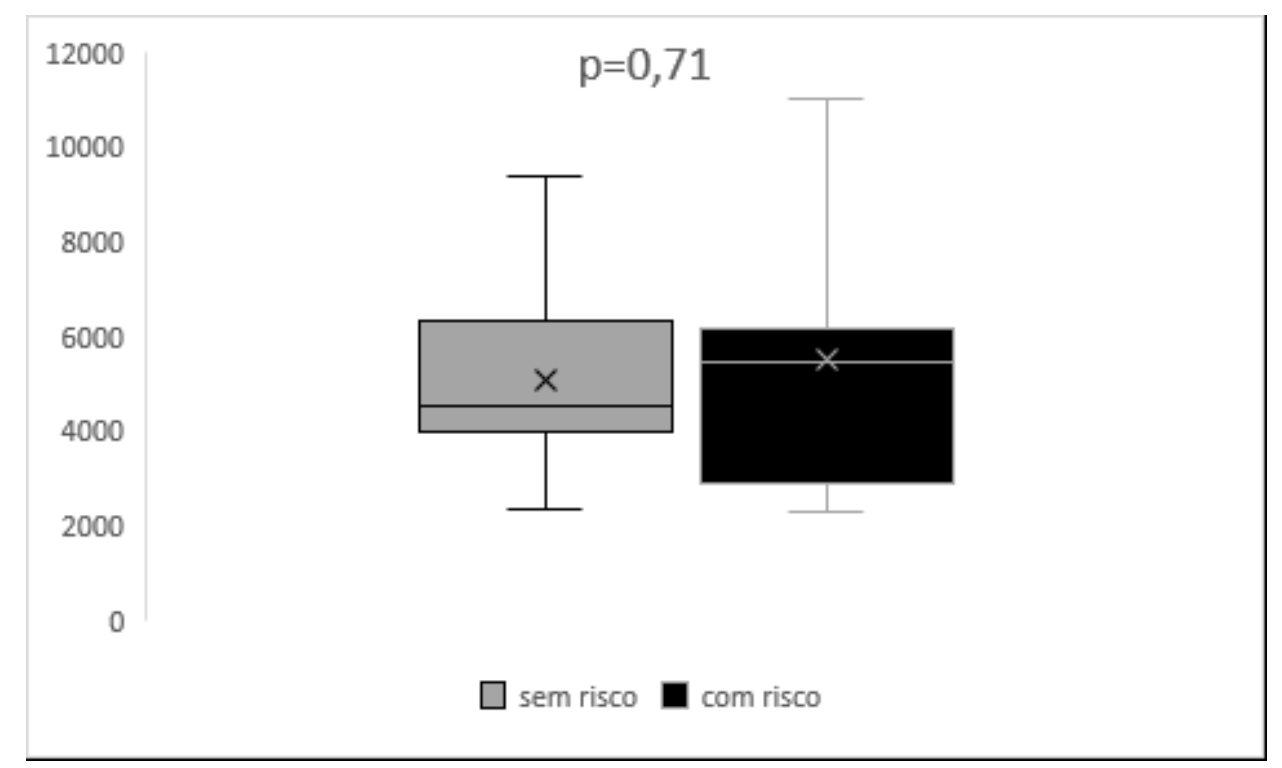

Figura 3. Comparação do nível de atividade física entre os sujeitos com e sem risco para o desenvolvimento de TMC

\section{DISCUSSÃO}

Não foram encontradas associações entre o NAF habitual e os TMC na amostra estudada. Além disso, não houve diferença da pontuação do SRQ-20 entre os sujeitos mais ativos (>6.000 passos/dia) e menos ativos (<6.000 passos/dia), 
bem como do NAF entre os sujeitos com risco ( $\geq 8$ questões) e sem risco ( $<8$ questões) para o desenvolvimento de TMC.

Tais dados vão ao encontro dos achados de Pie et al. ${ }^{13}$, no qual avaliaram 83 servidores universitários, e de Henriques et al. ${ }^{14}$, que incluíram 1520 mulheres, sendo 225 delas compreendendo a faixa-etária de 20 a 29 anos, e da mesma forma, não encontraram associações entre os TMC e o NAF. Cabe destacar que esses estudos ${ }^{13,14}$ utilizaram 0 Questionário Internacional de Atividade Física (IPAQ) como instrumento de avaliação do NAF, diferentemente do nosso trabalho que usou o pedômetro como método de avaliação.

Contudo, no estudo de Omena Silva e Neto ${ }^{15}$, foi observada associação entre o NAF (IPAQ), e os TMC (SRQ20), em 220 universitários da área de saúde de uma universidade pública. Além desta associação, foi encontrado que os estudantes inativos apresentaram três vezes mais chances de desenvolver TMC que os ativos. De forma semelhante, Rocha et al. ${ }^{16}$, em seu estudo com 3.597 indivíduos, encontraram que a prevalência de TMC (através do SRQ-20) foi menor entre os indivíduos ativos (sendo a atividade física classificada em leve, moderada ou pesada, conforme equivalentes metabólicos). Corroborando, Rios et al. ${ }^{17}$ incluíram, em seu estudo, 3.597 indivíduos, sendo 1.400 jovens com idade entre 15 e 29 anos, e identificaram uma associação negativa entre o NAF de lazer (mensurado através de questionário, sendo classificado em leve, moderado ou pesado) e os TMC (avaliado por meio do SRQ-20).

Cabe ressaltar que nos estudos de Rocha et al. ${ }^{16}$ e Rios et al. ${ }^{17}$ incluíram uma amostra significativamente maior que a do presente estudo, e esse fator pode explicar em parte os resultados distintos entre os estudos. Além disso, os estudos apresentados avaliaram a atividade física através de questionário, e o presente estudo optou por mensurar essa variável de uma forma mais objetiva e quantitativa, através do uso do pedômetro.

É importante destacar a lacuna de estudos que relacionam o desenvolvimento dos TMC com o NAF mensurado através do pedômetro, o que torna o estudo com caráter inovador, pois utilizou uma ferramenta objetiva para avaliar 0 nível NAF . Sob esse aspecto, McNamara, Hudson e Taylor ${ }^{18}$ reiteram que os pedômetros apresentam como vantagens o baixo custo e a praticidade, podendo ser utilizados como determinante do NAF em indivíduos jovens. Corroborando, 0 trabalho de Darvall, Parker e Story ${ }^{19}$ demonstraram a viabilidade e a aceitabilidade de uma intervenção de atividade física orientada pelo pedômetro. Ademais, O'Connell et al. ${ }^{20}$ encontraram que os pedômetros são precisos em sua sensibilidade de detecção de passo e são monitores válidos para a quantificação da atividade física.

Esses autores ainda relatam que a atividade física integra um estilo de vida saudável e apresenta benefício para a saúde física e mental, e uma contagem diária de 10 mil passos é recomendada globalmente para atingir um nível adequado de atividade física. No entanto, em nosso estudo, a média de passos das jovens avaliadas ficou abaixo desse ponto de corte $(5.318,14$ passos por dia), indicando um baixo nível de atividade física, constituindo um fator de risco para o desenvolvimento de doenças crônicas, como mostra o estudo de Tudor-Locke et al. ${ }^{21}$. Esses autores analisaram o comportamento de indivíduos jovens e encontraram probabilidades reduzidas de obesidade ou sobrepeso / obesidade 
relacionadas a cada incremento de 3.000 passos dados por dia. Ainda, em uma amostra de adultos, houve correlação negativa entre o número de passos diários com indicadores de saúde, como por exemplo, o percentual de gordura corporal, a circunferência da cintura e o índice de massa corporal22.

Apesar do presente estudo não encontrar associações entre o NAF e questões relativas à saúde mental, foi observado um perfil preocupante relacionado ao baixo NAF (5.318,14 passos) e uma pontuação indicativa de TMC (pontuação de 9,04 no SRQ-20) nas jovens avaliadas, indicando dois fatores de risco que podem afetar a qualidade de vida das mesmas.

Corroborando, Rios et al. ${ }^{17}$ também encontraram perfil semelhante, em que a maioria dos jovens investigados foram classificados como praticantes de atividade física leve, e a prevalência de TMC foi significativa entre jovens da mesma faixa-etária do presente estudo. Ainda, o trabalho realizado por Padovani et al. ${ }^{23}$ evidenciou que indicadores de estresse, burnout, ansiedade e depressão revelaram-se comuns em uma amostra formada por jovens acadêmicos, sendo a população feminina a mais vulnerável. Os autores também consideraram que o sofrimento psicológico pode ter implicações consideráveis no processo de aprendizagem e formação do futuro profissional.

Considerando o perfil encontrado em nosso estudo, é importante que ações preventivas sejam fomentadas no âmbito acadêmico, conforme exposto por Leite et al. ${ }^{24}$. Esses autores inferem também que os acadêmicos merecem atenção quanto aos aspectos relacionados à segurança, recursos financeiros, cuidados de saúde, oportunidades de recreação e lazer. Além disso, sabendo que as acadêmicas apresentam mais queixas de sofrimento mental, é relevante promover ações de cuidado e prevenção da saúde mental das mesmas ${ }^{6}$. Ademais, Silva e Costa $^{25}$ encontraram maior prevalência de TMC em mulheres acadêmicas do curso de fisioterapia, quando comparado a outros cursos da área, amostra essa estudada no presente estudo.

Durante a graduação, ações preventivas são fundamentais desde o início da vida acadêmica. As dificuldades e anseios na vida desses estudantes começam a aparecer devido a fatores que vão desde o medo de uma rotina nova que se inicia, até a ansiedade da finalização do curso e as exigências da sociedade quanto ao mercado de trabalho. Assim, com base nos resultados do presente estudo, e revelada a necessidade de políticas Universitárias que proporcionem apoio integral ao estudante, o estudo ressalta a importância da universidade em fomentar ações integradas de prevenção e tratamento do estudante universitário, bem como o estímulo a prática de atividade física.

\section{CONSIDERAÇÕES FINAIS}

Neste estudo, não foi possível constatar estatisticamente a relação entre o NAF e os TMC, porém demonstrou um grupo homogêneo, caracterizando a população acadêmica avaliada, que em sua grande maioria apresentou-se 
inativa e com indicativo de TMC. Este estudo chama a atenção para a importância de programas de atenção à saúde no meio acadêmico, incluindo ações que favoreçam a prática de atividade física de lazer, tendo em vista a contribuição do comportamento ativo na melhoria da saúde mental. Nota-se a necessidade de mais estudos que contemplem o universitário, de forma mais global, priorizando o corpo e mente deste futuro profissional da área da saúde. Ademais, sugere-se que mais estudos com esse enfoque sejam realizados com o público masculino, incluindo cursos de graduação de áreas diversas.

\section{REFERÊNCIAS}

1. Schleich ALR. Integração a educação superior e satisfação acadêmica de estudantes ingressantes e concluintes: um estudo sobre relações. 2006.

2. Ansolin AGA, Rocha DLB, Santos RPd, Dal Pozzo VC. Prevalência de transtorno mental comum entre estudantes de psicologia e enfermagem. Arquivos de Ciências da Saúde. 2015(3):42-5\%V 22.

3. Pinho R. Caracterização da clientela de um programa de atendimento psicológico a estudantes universitários. Psicología, Conocimiento y Sociedad. 2016;6(1):114-30.

4. Goldberg D, Huxley P. Commom mental disorders: a bio-social model. London: Tavistock; 1992.

5. Brasil. Cadernos de Atenção Básica - Saúde mental / Ministério da Saúde, Secretaria de Atenção à Saúde, Departamento de Atenção Básica, Departamento de AçõesProgramáticas Estratégicas. 2013;34:176.

6. Adamoli AN, Azevedo MR. Padrões de atividade física de pessoas com transtornos mentais e de comportamento. Ciência \& Saúde Coletiva. 2009;14:243-51.

7. Harding TW, De Arango V, Baltazar J, Climent CE, Ibrahim H, Ladrido-Ignacio L, et al. Mental disorders in primary health care: a study of their frequency and diagnosis in four developing countries. Psychological medicine. 1980;10(2):231-41. 
8. Mari JJ, Williams P. A validity study of a psychiatric screening questionnaire (SRQ-20) in primary care in the city of Sao Paulo. Br J Psychiatry. 1986;148:23-6.

9. Volcan SMA, Sousa PLR, Mari JdJ, Horta BL. Relação entre bem-estar espiritual e transtornos psiquiátricos menores: estudo transversal. Revista de Saúde Pública. 2003;37:440-5.

10. Colpani V, Spritzer PM, Lodi AP, Dorigo GG, Miranda IASd, Hahn LB, et al. Atividade física de mulheres no climatério: comparação entre auto-relato e pedômetro. Revista de Saúde Pública. 2014;48:258-65.

11. Bassett DR, Jr., Toth LP, LaMunion SR, Crouter SE. Step Counting: A Review of Measurement Considerations and Health-Related Applications. Sports Med. 2017;47(7):1303-15.

12. Lara S, Copetti J, Lanes KG, Puntel RL, Folmer V. Imagem corporal, medidas antropométricas e atividade física como ferramentas para a educação em saúde no contexto escolar. Revista Ciências \& Ideias. 2014;4(2):27-46.

13. Pie A, Pinto L, Rocha S, Cardoso J, Amorim C, Carneiro L. Nível de atividade física e transtornos mentais comuns entre trabalhadores de uma instituição de ensino superior da Bahia. Arq Cien Esp. 2012;1(1):46-53.

14. Henriques IF, Walsh IAPd, Meirelles MCCC, Pereira GdA, Castro SSd. Relation of common mental disorder, physical activity and body mass index in women, population-based study. Journal of Physical Education. 2017;28.

15. Omena Silva A, Neto JLC. Associação entre níveis de atividade física e transtorno mental comum em estudantes universitários/Association between levels of physical activity and common mental disorder in university students. Motricidade. 2014;10(1):49.

16. Rocha SV, Araújo TMd, Almeida MMGd, Virtuoso Júnior JS. Prática de atividade física no lazer e transtornos mentais comuns entre residentes de um município do Nordeste do Brasil. Rev Bras Epidemiol. 2012;15(4):871-83.

17. Rios LC, Almeida MMGd, Rocha SV, Araújo TM, Pinho PdS. Atividades físicas de lazer e transtornos mentais comuns em jovens de Feira de Santana, Bahia. Revista de Psiquiatria do Rio Grande do Sul. 2011;33:98-102. 
18. McNamara E, Hudson Z, Taylor SJ. Measuring activity levels of young people: the validity of pedometers. Br Med Bull. 2010;95:121-37.

19. Darvall JN, Parker A, Story DA. Feasibility and acceptability of remotely monitored pedometer-guided physical activity. Anaesth Intensive Care. 2016;44(4):501-6.

20. O'Connell S, ÓLaighin G, Kelly L, Murphy E, Beirne S, Burke N, et al. These Shoes Are Made for Walking: Sensitivity Performance Evaluation of Commercial Activity Monitors under the Expected Conditions and Circumstances Required to Achieve the International Daily Step Goal of 10,000 Steps. PLoS ONE. 2016;11(5):e0154956.

21. Tudor-Locke C, Craig CL, Cameron C, Griffiths JM. Canadian children's and youth's pedometer-determined steps/day, parent-reported TV watching time, and overweight/obesity: The CANPLAY Surveillance Study. The International Journal of Behavioral Nutrition and Physical Activity. 2011;8:66.

22. Pillay JD, van der Ploeg HP, Kolbe-Alexander TL, Proper KI, van Stralen M, Tomaz SA, et al. The association between daily steps and health, and the mediating role of body composition: a pedometer-based, cross-sectional study in an employed South African population. BMC Public Health. 2015;15(1):174.

23. Padovani RdC, Neufeld CB, Maltoni J, Barbosa LNF, Souza WFd, Cavalcanti HAF, et al. Vulnerabilidade e bem-estar psicológicos do estudante universitário. Revista Brasileira de Terapias Cognitivas. 2014;10(1):02-10.

24. Leite ACB, Grillo LP, Caleffi F, Mariath AB, Stuker H. Qualidade de vida e condições de saúde de acadêmicos de nutrição. Espaço para a Saúde-Revista de Saúde Pública do Paraná. 2012;13(1):82-90.

25. Silva RS, Costa LA. Prevalência de transtornos mentais comuns entre estudantes universitários da área da saúde. Encontro: Revista de Psicologia. 2015;15(23):105-12.2. 